\title{
Crack hands and crack thumbs in chronic cocaine abuse
}

\author{
Aaqib Aslam', Shagufta Rather', Arshid Hussain², Iffat Hassan', Mahvish Renzu³ \\ ${ }^{1}$ Department of Dermatology, Government Medical College Srinagar, Jammu and Kashmir, India, ${ }^{2}$ Department of \\ Psychiatry, Government Medical College Srinagar, Jammu and Kashmir, India, ${ }^{3}$ Acharya Shri Chander College of Medical \\ Sciences Jammu and Kasmir, India
}

Corresponding author: Dr. Aaqib Aslam, E-mail: shahaaqib2010@gmail.com

Cocaine, a substance very commonly abused around the world, is a stimulant that increases dopamine concentrations in the brain reward system, producing a dose-dependent feeling of euphoria, which leads to long-term alterations in neuronal pathways, ultimately giving rise to addiction [1].

Crack cocaine is a cheaper and more potent version of cocaine prevalent in lower socioeconomic classes. Numerous dermatological manifestations of cocaine abuse have recently been mentioned, including pyoderma gangrenosum and Raynaud's phenomenon, to name a few [1-3]. We report the case of a 38-year-old male chronically abusing crack cocaine for the past 5 years, displaying blackened punctiform hyperkeratotic lesions on the palms and the ventral surfaces of the fingers, a condition known as crack hands [2]. These lesions are more evident in the dominant hand, which, in this case, is the left hand (Fig. 1). Burns on the hands along with callus or blister formations on the thumb are due to the act of igniting cocaine pipe lighters, the latter known as a crack thumb [3]. Such blister formation can, indeed, be seen in this case of chronic cocaine abuse (Fig. 2).

\section{Consent}

The examination of the patient was conducted according to the principles of the Declaration of Helsinki.

The authors certify that they have obtained all appropriate patient consent forms, in which the patients have given consent for images and other clinical information to be included in the journal. The patients understand that their names and initials will not be published and due effort will be made to conceal their identity, but that anonymity cannot be guaranteed.

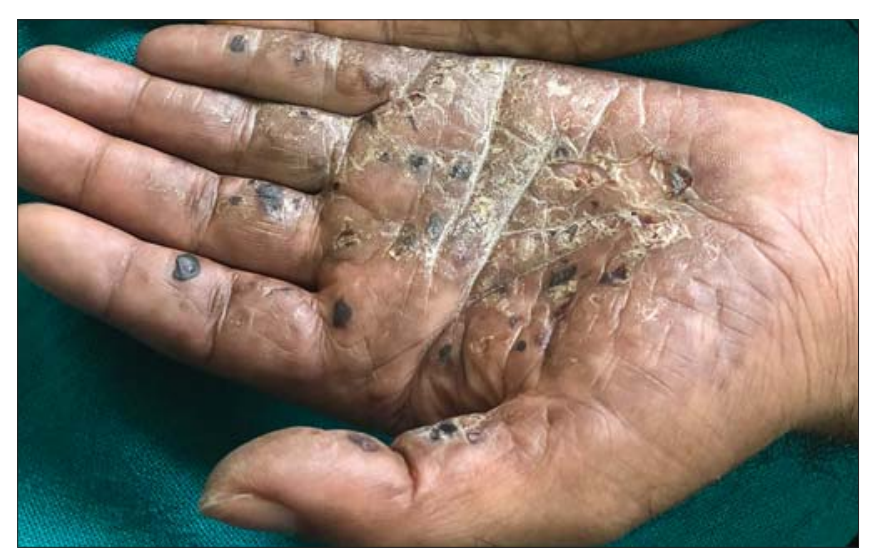

Figure 1: Blackened punctiform hyperkeratotic lesions located on the palms and the ventral surfaces of the fingers known as crack hands.

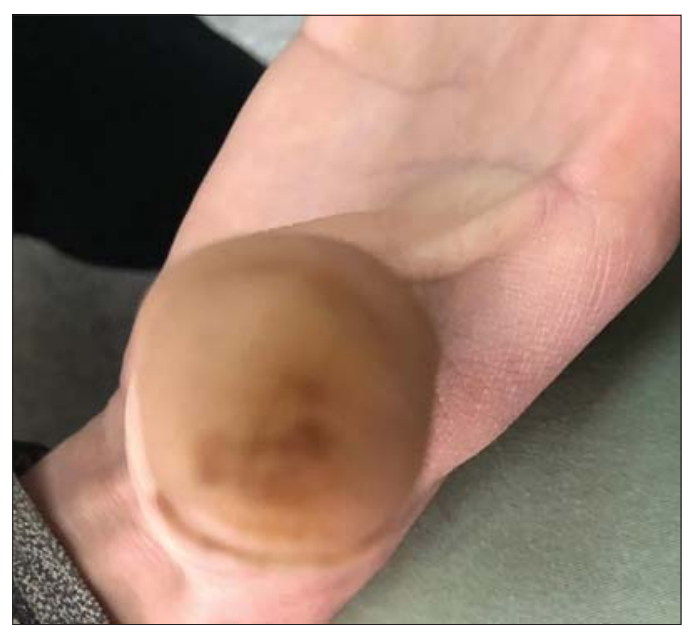

Figure 2: A blister on the thumb known as a crack thumb.

\section{REFERENCES}

1. Moreno-Ar tero E, Querol-Cisneros E, Rodriguez-Garijo N, Tomas-Velazquez A, Antonanzas J, Secundino F, et al. Mucocutaneous manifestations of cocaine abuse: a review. J Eur

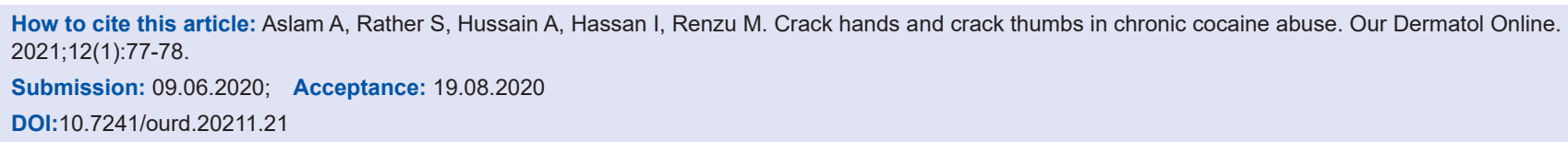


www.odermatol.com

Acad Dermatol Venereol. 2018;32:1420-6.

2. Incel Uysal P, Gur Aksoy G, Yalcin B. Clinical fi ndings and outcomes in patients with pyoderma gangrenosum: a single tertiary centre experience. Our Dermatol Online. 2019;10:17-22.

3. Chang P, Vásquez Acajabón MV, Anzueto E. Raynaud's phenomenon. Our Dermatol Online. 2019;10:94-7.
Copyright by Aaqib Aslam, et al. This is an open access article distributed under the terms of the Creative Commons Attribution License, which permits unrestricted use, distribution, and reproduction in any medium, provided the original author and source are credited.

Source of Support: Nil, Conflict of Interest: None declared. 